\title{
Editorial
}

\section{Tuberculosis: novel approaches to an old disease}

\author{
Giovanni Fadda
}

Catholic University of the Sacred Heart, Rome, Italy

Key words: tuberculosis, Mycobacterium

J Infect Dev Ctries 2012; 6(1):4-5.

(Received and Accepted 07 January 2012)

Copyright (C) 2012 Fadda. This is an open-access article distributed under the Creative Commons Attribution License, which permits unrestricted use, distribution, and reproduction in any medium, provided the original work is properly cited

Tuberculosis (TB), one of the most ancient and deadly diseases of mankind, has ravaged human communities since the beginning of civilization, and still remains one of the most devastating infectious diseases in the $21^{\text {st }}$ century. The latest report released by the World Health Organization estimates that 1.45 million deaths each year are due to TB, with 9 million incident cases and 14 million prevalent cases (WHO, Global tuberculosis control 2011, http://www.who.int/tb/publications/global_report/en/i ndex.html).

Most TB cases occur in developing countries, with Africa and some south-east Asian countries bearing most of the burden ( $>300$ TB cases/100,000 population), though high $\mathrm{TB}$ rates are observed also in China, Russia, eastern European countries and South America. TB is also a disease that targets young adults and as such it also creates a main obstacle towards a sound economic and social development of the countries where it is endemic.

Nevertheless, TB is a curable disease that has been effectively controlled in many parts of the world since the middle of the $20^{\text {th }}$ century through the implementation of effective, yet simple, control measures, diagnostics, and the use of long-lasting drug regimens, and as a result $\mathrm{TB}$ rates in western countries are below $5 \mathrm{~TB}$ cases/100,000 population.

Since the WHO in 1993 declared TB a global emergence, significant efforts have been made to tackle TB epidemics in developing countries by investing in infrastructure, building new laboratories, and training people to diagnose and care for TB patients. The aim is to make available to all countries traditional diagnostic tools, such as culturing bacilli and performing phenotypic drug susceptibility testing, as well as more recent ones such as molecular systems for the direct detection of Mycobacterium tuberculosis in clinical specimens, rapid identification of drug resistance genotypes, or the introduction of the interferon- $\gamma$ release assays for the detection of TB infection. Nevertheless, these efforts have been inadequate so far and more resources must be devoted to the battle against $\mathrm{TB}$ if a significant improvement is to be made. Government agencies, health authorities, medical doctors and scientists face many other challenges in addition to limited resources, including the following:

- The TB epidemic is in many parts of the world linked to the HIV epidemic, which worsens the clinical outcome of TB and facilitates bacilli transmission, particularly in the young adult population.

- The emergence of multi-drug resistant $M$. tuberculosis strains (MDR-TB), which are strains resistant to the first-line drugs isoniazid and rifampin, and more recently the emergence of extensively drug-resistant strains (XDR-TB), which are MDR-TB strains resistant also to any fluoroquinolone and to at least one of the three injectable drugs used for TB treatment (amikacin, capreomycin and kanamycin), complicates treatment. The lack of other effective drugs against TB makes the treatment of patients with XDR-TB challenging and high mortality rates are observed. The spread of these strains in other parts of the world is seen as a major threat and many efforts are being made to attempt to reduce the risk of transmission.

- The lack of new drugs is a major problem. Unfortunately, for too many years, pharmaceutical 
companies have not invested in the development of new drugs against TB and only in the last two decades, thanks to government-funded agencies and contributions from philanthropic foundations, has it been possible to develop new drugs, some of which are completing clinical trials and may be available in the near future.

- The lack of an effective vaccine against TB is another challenge. The ninety-year-old Bacille Calmette and Guerin (BCG) strain is the only vaccine available against TB. Although it is widely used in all countries where TB is endemic, where it is administered immediately after birth because of its efficacy in preventing the most dangerous form of $\mathrm{TB}$ in infancy, $\mathrm{BCG}$ is ineffective against active TB in adults and as a result is not effective in preventing transmission. The availability of a new and improved vaccine against TB would provide a powerful tool to control TB, but there is still a long road ahead before any new vaccine will be used. The lack of immune correlates of protection for $\mathrm{TB}$, the fact that evaluation of new vaccines in pre-clinical studies have shown only partial improvement over BCG and the fact that the phase 3 efficacy trials will last many years, make the goal of acquiring a new vaccine against TB in the near future very complicated.

The declaration of the global emergence of TB prompted many countries to drastically increase funds for TB-related research, including the development of new diagnostics, vaccines and drugs, and though there has not yet been a clear breakthrough, the impact of this new interest in a neglected disease has been relevant. In fact, in the last ten years, many research groups have established laboratories and facilities in countries where TB is endemic with the aim to implement clinical studies for the evaluation of new drugs and vaccines. These state-of-the-art facilities are fully equipped with the most advanced diagnostic tools and as a result they are already providing excellent support for TB control programs in these resource-limited countries. These advances have allowed local health authorities and international health organizations to implement effective TB control programs, granting access for many people to the most effective control tools against TB. This renewed interest in TB is also a great opportunity at the global level to control the disease and it is a clear example of how funneling significant resources into research can improve control programs. The results can be immediate and the reward in terms of lives saved greater than expected.

This issue of the Journal of Infection in Developing Countries is dedicated to tuberculosis research with several related papers on different aspects of the disease in developing countries. It is our way to contribute to the fight against this global threat.

\section{Corresponding author}

Giovanni Fadda

Institute of Microbiology

A. Gemelli University Hospital

Catholic University of the Sacred Heart

Rome, Italy

Telephone: ++390630154964

Email: giovannifadda@rm.unicatt.it 